\title{
Classical Physics of Thermal Scalar Radiation in Two Spacetime Dimensions
}

\author{
Timothy H. Boyer \\ Department of Physics, City College of the City \\ University of New York, New York, New York 10031
}

\begin{abstract}
Thermal scalar radiation in two spacetime dimensions is treated within relativistic classical physics. Part I involves an inertial frame where are given the analogues both of Boltzmann's derivation of the Stefan-Boltzmann law and also Wien's derivation of the displacement theorem

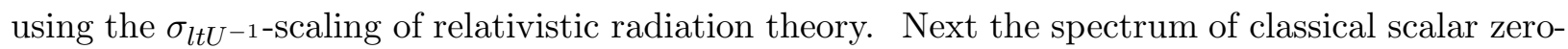
point radiation in an inertial frame is derived both from $\sigma_{l t U^{-1}-\text { scale invariance and from Lorentz }}$ invariance. Part II involves the behavior of thermal radiation in a coordinate frame undergoing (relativistic) constant acceleration, a Rindler frame. The radiation normal modes in a Rindler frame are obtained. The classical zero-point radiation of inertial frames is transformed over to the coordinates of a Rindler frame. Although for zero-point radiation the two-field correlation function at different spatial points at a single time is the same between inertial and Rindler frames, the correlation function at two different times at a single Rindler spatial coordinate is different, and has a natural extension to non-zero temperature. The thermal spectrum in the Rindler frame is then transferred back to an inertial frame, giving the familiar Planck spectrum.
\end{abstract}




\section{INTRODUCTION}

Thermal radiation holds an unusual place in physics. Although thermal radiation provides a simple system for the discussion of thermodynamics, the determination of the spectrum of thermal radiation is often regarded as an intractable problem within classical physics. Here we provide a discussion of thermal radiation within classical physics which is different from the historical treatments. Rather than attempting to determine the thermal spectrum from use of nonrelativistic classical statistical mechanics or from scattering by nonrelativistic mechanical systems, we use the scaling symmetries of relativistic classical radiation both in inertial frames and in (relativistic) accelerating coordinate frames. The Planck spectrum arises naturally from ideas of classical radiation equilibrium. The analysis also suggests that relativistic physics is important in problems of classical radiation equilibrium.

The present article is at the interface between pedagogy and research. In the first part, we review some of the ideas of thermal radiation in inertial frames which appear in text books of thermodynamics[1] and of modern physics[2] but in the simpler context of scalar radiation in two spacetime dimensions. We give the analogues of Boltzmann's derivation of the Stefan-Boltzmann law [3] and Wein's derivation of the displacement theorem[4]. However, the analysis also emphasizes aspects which are rarely treated in the text books, such as the

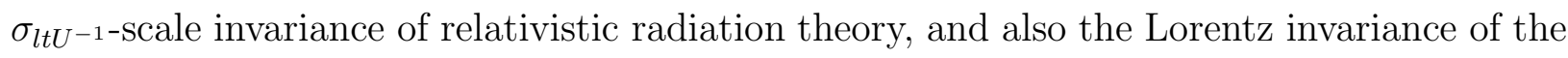
radiation spectrum which assigns to each normal mode an energy equal to a constant times the frequency. In the second part we introduce a Rindler coordinate frame corresponding to a system accelerating relative to an inertial frame. Now a Rindler frame is a more complicated system than an inertial frame and is usually introduced in connection with general relativity. [5] However, a Rindler frame actually involves no more than the ideas

of special relativity. [6] Since classical radiation transforms as a tensor, we expect that classical zero-point radiation will reappear in any other coordinate frame, including a Rindler frame. Scalar radiation has trivial tensor transformations and so is easily transferred to the coordinates of the Rindler frame. The radiation normal modes in the Rindler frame are introduced as a natural generalization of the radiation modes in an inertial frame. In a Rindler frame, there is a natural extension of the radiation correlation function from zeropoint radiation over to thermal radiation. Finally we show how to bring the information about the spectrum of thermal radiation in a Rindler frame back to an inertial frame. 
The analysis presented here in the simple case of scalar radiation and two spacetime dimensions involves only elementary functions [7] and extensions of familiar ideas. Nevertheless, the conclusions are radically different from what appears in current text books of modern physics. The present analysis emphasizes the relativistic nature of the thermal radiation of a relativistic field.

\section{PART I: THERMAL RADIATION IN AN INERTIAL FRAME}

\section{A. Simplified Model Theory}

\section{Basic Assumptions}

Our discussion of radiation equilibrium begins with certain fundamental assumptions based upon experimental observations. 1) We assume that we are dealing with a relativistic radiation field so that the speed of light in vacuum $c$ enters as a fundamental constant. 2) We assume that radiation equilibrium exists in the form corresponding to Stefan's law for electromagnetic radiation, $u_{T}=\sigma_{S} T^{4}$, where $u_{T}$ is the thermal energy density per unit volume, $T$ is the absolute temperature, and $\sigma_{S}$ (Stefan's constant) is a fundamental constant. 3) We assume there is random radiation present at the absolute zero of temperature. 4) We assume that thermal equilibrium exists in a uniformly accelerated coordinate frame or (by the equivalence principle) in a gravitational field.

The assumptions we have made are taken from experimental observations. Maxwell's equations for the electromagnetic field were developed in the 1860s based upon the electromagnetic observations of Ampere, Faraday, and their contemporaries. These equations are relativistically invariant and contain the speed of light in vacuum $c$. The development of thermodynamics also occurred during the nineteenth century and Stefan's observations [8] regarding thermal radiation were made in the 1870's. The idea of zero-point radiation arose during the twentieth century and the experimental observations of Spaarnay, Lamoreau and others [9] confirm the calculations based upon zero-point radiation. [10] Finally, it is a natural extension of our thermodynamics observations to assume that thermodynamics ideas hold in gravitational fields. Such ideas were used in the 1870s by Boltzmann in his derivation of the Maxwell velocity distribution for thermal particle velocities. [11] 


\section{Scalar Radiation in Two Spacetime Dimensions}

In this article, we wish to simplify the mathematics as much as possible while exploring the implications of thermal radiation. Therefore we will not treat the electromagnetic field in four spacetime dimensions (three space and one time dimension), but rather will discuss a model calculation involving scalar radiation in two spacetime dimensions (one space and one time dimension). [12] Instead of the six fields $E_{x}, E_{y}, E_{z}, B_{x}, B_{y}, B_{z}$ of electromagnetic theory satisfying Maxwell's equations in $(c t, x, y, z)$, we will consider only one relativistic scalar field $\phi$ which is a function of $(c t, x)$ in an inertial frame with spacetime metric $d s^{2}=g_{\mu \nu} d x^{\mu} d x^{\nu}$ where the indices $\mu$ and $\nu$ run over 0 and $1, x^{0}=c t, x^{1}=x$,

$$
d s^{2}=c^{2} d t^{2}-d x^{2}
$$

The behavior of the field $\phi$ follows from the Lagrangian density $\mathcal{L}=(1 / 8 \pi) \partial^{\mu} \phi \partial_{\mu} \phi$ corresponding to[13]

$$
\mathcal{L}=\frac{1}{8 \pi}\left[\frac{1}{c^{2}}\left(\frac{\partial \phi}{\partial t}\right)^{2}-\left(\frac{\partial \phi}{\partial x}\right)^{2}\right]
$$

and the wave equation $\partial_{\mu}\left[\partial \mathcal{L} / \partial\left(\partial_{\mu} \phi\right)\right]=0$ for the field is

$$
\frac{1}{c^{2}}\left(\frac{\partial^{2} \phi}{\partial t^{2}}\right)-\left(\frac{\partial^{2} \phi}{\partial x^{2}}\right)=0
$$

The associated stress-energy-momentum tensor density is given by $\mathcal{T}^{\mu \nu}=\left[\partial \mathcal{L} / \partial\left(\partial_{\mu} \phi\right)\right] \partial^{\nu} \phi-$ $g^{\mu \nu} \mathcal{L}$ so that the energy density $u=\mathcal{T}^{00}$ is

$$
\mathcal{T}^{00}=-\mathcal{T}^{11}=\frac{1}{8 \pi}\left[\frac{1}{c^{2}}\left(\frac{\partial \phi}{\partial t}\right)^{2}+\left(\frac{\partial \phi}{\partial x}\right)^{2}\right]
$$

and the momentum density is

$$
\mathcal{T}^{01}=\mathcal{T}^{10}=\frac{1}{4 \pi c} \frac{\partial \phi}{\partial t} \frac{\partial \phi}{\partial x}
$$

The energy $U$ in the field in a one-dimensional box extending from $x=a$ to $x=b$ follows as

$$
U=\int_{a}^{b} d x \frac{1}{8 \pi}\left[\frac{1}{c^{2}}\left(\frac{\partial \phi}{\partial t}\right)^{2}+\left(\frac{\partial \phi}{\partial x}\right)^{2}\right]
$$




\section{B. Thermodynamics of Radiation in a One-Dimensional Box}

The thermodynamics of radiation in a box (in this case a box with one spatial dimension) can be found in a traditional fashion from

$$
T d S=d U_{T}+p_{T} d V
$$

In the present case of one spatial dimension, the length $L$ will replace the volume $V$. Also, for a large box, we can neglect the (Casimir) effects[10] due to the discrete nature of the normal modes so that the energy density in an inertial frame is uniform throughout the box and the energy density is a function of temperature alone so that the thermodynamic equation (7) becomes

$$
T d S=d\left(u_{T} L\right)+p_{T} d L=L d u_{T}+\left(u_{T}+p_{T}\right) d L
$$

From the tensor equation (4) above, we see that the energy density and stress tensor density are equal in magnitude $T^{00}=-T^{11}$ so that the pressure $p_{T}=u_{T}$, and Eq. (8) becomes

$$
T d S=T\left(\frac{\partial S}{\partial T}\right)_{L} d T+T\left(\frac{\partial S}{\partial L}\right)_{T} d L=L \frac{d u_{T}}{d T} d T+2 u_{T} d L
$$

This gives us

$$
\left(\frac{\partial S}{\partial T}\right)_{L}=\frac{L}{T} \frac{d u_{T}}{d T}
$$

and

$$
\left(\frac{\partial S}{\partial L}\right)_{T}=\frac{2 u_{T}}{T}
$$

The equality of the mixed partials $\partial^{2} S /(\partial T \partial L)=\partial^{2} S /(\partial L \partial T)$ then implies

$$
\frac{\partial}{\partial L}\left(\frac{L}{T} \frac{d u_{T}}{d T}\right)=\frac{\partial}{\partial T}\left(\frac{2 u_{T}}{T}\right)
$$

or

$$
\frac{1}{T} \frac{d u_{T}}{d T}=\frac{2}{T} \frac{d u_{T}}{d T}-\frac{2 u_{T}}{T^{2}}
$$

with solution

$$
u_{T}=\sigma_{2 D} T^{2} \quad U_{T}=\sigma_{2 D} T^{2} L
$$

The entropy $S$ follows from Eq. (11) as

$$
S=2 \sigma_{2 D} T L
$$

Thus we have found for a one-dimensional box the thermodynamic expressions exactly analogous to Boltzmann's familiar expressions for a three-dimensional box. The constant $\sigma_{2 D}$ is analogous to Stefan's constant $\sigma_{S}$ for electromagnetic radiation in three-dimensional space. 


\section{Scaling and Fundamental Constants}

\section{1. $\sigma_{l t U^{-1}-S c a l i n g}$ for Any Radiation Theory Allowing Thermal Equilibrium}

Classical mechanics has no fundamental constants and accordingly allows separate scalings in length, time, and energy. Thus, for example, in principle, any classical mechanical system can be made twice as large, can perform at three times the speed, and involve four times the energy. However, a classical theory which contains fundamental constants has its scaling constrained by these fundamental constants. This situation is familiar in connection with the fundamental constant $c$ which enters relativistic systems. The fundamental constant $c$ involves length $l$ divided by time $t$ and so couples together the scalings of length and time so as to preserve the value of the fundamental constant $c$. In the paragraph above, we have seen that if thermal radiation equilibrium exists, then there must be a fundamental constant corresponding to Stefan's constant $\sigma_{S}$, which in our two-dimensional case is $\sigma_{2 D}$. The fundamental constant $\sigma_{S}$ (or in our case $\sigma_{2 D}$ ) couples together energy and length appearing in the thermal energy density $u_{T}$ and the thermal energy $k_{B} T$. Thus for any relativistic classical radiation theory which allows thermal equilibrium, the fundamental constants allow only those scalings which couple together length, time, and energy: length scales as $l \rightarrow l^{\prime}=\sigma l$, time scales as $t \rightarrow t^{\prime}=\sigma t$, and energy scales as $U \rightarrow U^{\prime}=U / \sigma$, for

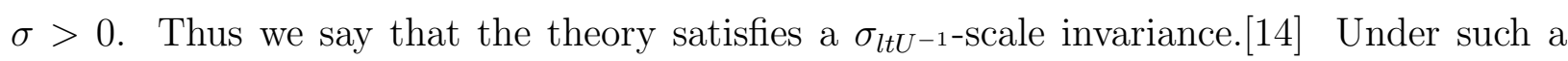
scaling, the fundamental constants $c$ and $\sigma_{2 D}$ are carried into themselves, the wave equation (3) is carried into itself, and solutions of the differential equation are carried into solutions.

\section{2. $\sigma_{l t U^{-1}-S c a l i n g}$ and Adiabatic Change in an Inertial Frame}

For a relativistic classical radiation theory which contains no fundamental lengths, a

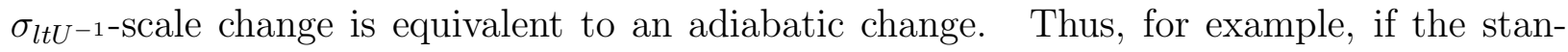

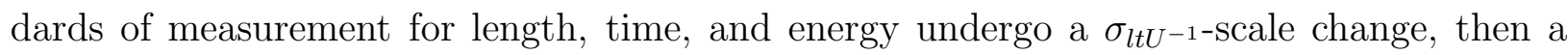
thermodynamic system will be interpreted as having a new temperature $T / \sigma$, a new box length $\sigma L$, and new energy $U / \sigma$. However, the entropy $S$ of the system in Eq. (15) is invariant under such a scale change. These changes are exactly the same as though one had

carried out an adiabatic compression or expansion of the radiation in the box. Under an adiabatic change, there is no change in the entropy of the system. Accordingly, equations 
(7) and (8) become

$$
0=d U_{T}+p_{T} d L=d U_{T}+\frac{U_{T}}{L} d L
$$

or

$$
U_{T}=\frac{\text { const }}{L}
$$

during an adiabatic change. But this equation (17) is invariant under a $\sigma_{l t U^{-1} \text {-scale change }}$ since $U_{T} \rightarrow U_{T} / \sigma$ while $L \rightarrow \sigma L$. Indeed the thermodynamics equations (14) and (15) transform under adiabatic change just as though the standards of measurement for length, time, and energy had undergone a $\sigma_{l t U^{-1} \text {-scale change. }}$

Although the theoretical calculation of Stefan's law[3] (comparable to our calculation here) was carried through by Boltzmann in the 1880s and is thoroughly familiar to many

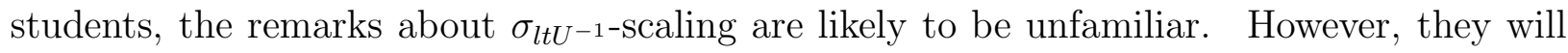
be useful in our later work.

\section{Radiation Spectrum in a Box}

\section{Radiation Normal Modes}

The radiation in a box can be described by a complete set of either standing waves or running waves with appropriate wave vectors. In the present case, we will choose standing wave solutions which vanish at the walls $x=a$ and $x=b$ of the box (Dirichlet boundary conditions) so that a normalized normal mode can be written as

$$
\phi_{n}(c t, x)=f_{n}\left(\frac{2}{b-a}\right)^{1 / 2} \sin \left[\frac{n \pi}{b-a}(x-a)\right] \cos \left[\frac{n \pi}{b-a} c t+\theta_{n}\right]
$$

Then the radiation field in the box can be written as a sum over all the normal modes

$$
\phi(c t, x)=\sum_{n=1}^{\infty} f_{n}\left(\frac{2}{b-a}\right)^{1 / 2} \sin \left[\frac{n \pi}{b-a}(x-a)\right] \cos \left[\frac{n \pi}{b-a} c t+\theta_{n}\right]
$$


where $\theta_{n}$ is an appropriate phase. From Eq. (5), we find that each mode $\phi_{n}(c t, x)$ has the time-average spatial energy density

$$
\begin{aligned}
u_{n}(x) & =\left\langle\frac{1}{8 \pi}\left[\frac{1}{c^{2}}\left(\frac{\partial \phi_{n}}{\partial t}\right)^{2}+\left(\frac{\partial \phi_{n}}{\partial x}\right)^{2}\right]\right\rangle_{\text {time }} \\
& =\frac{1}{8 \pi}\left(\frac{n \pi}{b-a}\right)^{2} f_{n}^{2} \frac{2}{b-a}\left\{\sin ^{2}\left[\frac{n \pi}{b-a}(x-a)\right]\left\langle\sin ^{2}\left[\frac{n \pi}{b-a} c t+\theta_{n}\right]\right\rangle_{\text {time }}\right. \\
& \left.+\cos ^{2}\left[\frac{n \pi}{b-a}(x-a)\right]\left\langle\cos ^{2}\left[\frac{n \pi}{b-a} c t+\theta_{n}\right]\right\rangle_{t i m e}\right\} \\
& =\frac{1}{8 \pi}\left(\frac{n \pi}{b-a}\right)^{2} \frac{f_{n}^{2}}{b-a}
\end{aligned}
$$

which is uniform in space. The total mode energy $U_{n}$ found by integrating over the length is given by

$$
U_{n}=\frac{1}{8 \pi}\left(\frac{n \pi}{b-a}\right)^{2} f_{n}^{2}
$$

\section{Two-Point Correlation Function for Random Radiation}

Coherent radiation involves fixed phase relations $\theta_{n}-\theta_{n^{\prime}}$ between the various modes $\phi_{n}$ and $\phi_{n^{\prime}}$ which are used to decompose a radiation pattern. Random radiation, such as is involved in thermal radiation, involves the opposite situation. Random radiation can be written in the form of Eq. (19) where the phases $\theta_{n}$ are randomly distributed on the interval $[0,2 \pi)$ and are independently distributed for each $n$. It is convenient to characterize random radiation by taking the two-point correlation function of the fields $\left\langle\phi(c t, x) \phi\left(c t^{\prime}, x^{\prime}\right)\right\rangle$ obtained by averaging over the random phases as

$$
\begin{gathered}
\left\langle\cos \theta_{n} \cos \theta_{n^{\prime}}\right\rangle=\left\langle\sin \theta_{n} \sin \theta_{n^{\prime}}\right\rangle=(1 / 2) \delta_{n, n^{\prime}} \\
\left\langle\cos \theta_{n} \sin \theta_{n^{\prime}}\right\rangle=0
\end{gathered}
$$


The two-point correlation function for a general distribution of random classical scalar waves is found by averaging over the random phases $\theta_{n}[15]$

$$
\begin{aligned}
\left\langle\phi(c t, x) \phi\left(c t^{\prime}, x^{\prime}\right)\right\rangle & =\mathrm{AV} \sum_{n=1}^{\infty} f_{n}\left(\frac{2}{b-a}\right)^{1 / 2} \sin \left[\frac{n \pi}{b-a}(x-a)\right] \cos \left[\frac{n \pi}{b-a} c t+\theta_{n}\right] \\
& \times \sum_{n^{\prime}=1}^{\infty} f_{n^{\prime}}\left(\frac{2}{b-a}\right)^{1 / 2} \sin \left[\frac{n^{\prime} \pi}{b-a}\left(x^{\prime}-a\right)\right] \cos \left[\frac{n^{\prime} \pi}{b-a} c t^{\prime}+\theta_{n^{\prime}}\right] \mathrm{AV} \\
& =\sum_{n=1}^{\infty} \frac{f_{n}^{2}}{b-a} \sin \left[\frac{n \pi}{b-a}(x-a)\right] \sin \left[\frac{n \pi}{b-a}\left(x^{\prime}-a\right)\right] \cos \left[\frac{n \pi}{b-a} c\left(t-t^{\prime}\right)\right]
\end{aligned}
$$

Since we are not interested in the discrete mode structure of the box but rather in the large-box limit, it is convenient to use the identity $2 \sin A \sin B=\cos (A-B)-\cos (A+B)$ to rewrite the correlation function in Eq. (24) as

$$
\begin{aligned}
\left\langle\phi(c t, x) \phi\left(c t^{\prime}, x^{\prime}\right)\right\rangle & =\frac{1}{2} \sum_{n=1}^{\infty} \frac{f_{n}^{2}}{b-a} \cos \left[\frac{n \pi}{b-a}\left(x-x^{\prime}\right)\right] \cos \left[\frac{n \pi}{b-a} c\left(t-t^{\prime}\right)\right] \\
& -\frac{1}{2} \sum_{n=1}^{\infty} \frac{f_{n}^{2}}{b-a} \cos \left[\frac{n \pi}{b-a}\left(x+x^{\prime}-2 a\right)\right] \cos \left[\frac{n \pi}{b-a} c\left(t-t^{\prime}\right)\right]
\end{aligned}
$$

We are interested only in points $x$ and $x^{\prime}$ which are far from the edges of a large box, and so we will drop the term involving the large separation $\left(x+x^{\prime}-2 a\right)$ where the cosine function is oscillating very rapidly. Also, for $b-a$ large (corresponding to a large box), we will replace the sum by an integral $\sum \rightarrow \int d n$, and will write $k=n \pi /(b-a)$ so that

$$
\begin{aligned}
\left\langle\phi(c t, x) \phi\left(c t^{\prime}, x^{\prime}\right)\right\rangle & =\frac{1}{2} \int_{0}^{\infty} d n \frac{f_{n}^{2}}{b-a} \cos \left[\frac{n \pi}{b-a}\left(x-x^{\prime}\right)\right] \cos \left[\frac{n \pi}{b-a} c\left(t-t^{\prime}\right)\right] \\
& =\frac{1}{2 \pi} \int_{0}^{\infty} d k f^{2}(k) \cos \left[k\left(x-x^{\prime}\right)\right] \cos \left[k c\left(t-t^{\prime}\right)\right] \\
& =\frac{1}{4 \pi} \int_{-\infty}^{\infty} d k f^{2}(|k|) \cos \left[k\left(x-x^{\prime}\right)-|k| c\left(t-t^{\prime}\right)\right]
\end{aligned}
$$

where in the last line we have used the identity $2 \cos A \cos B=\cos (A+B)+\cos (A-B)$ and have incorporated the two cosine terms by adding the integral over negative values of $k$. The connection between the spectral function $f^{2}(|k|)$ and the energy $U(k)$ of a normal mode of wave number $k$ follows from Eq. (21) as

$$
U(k)=\frac{1}{8 \pi} k^{2} f^{2}(|k|)
$$




\section{E. Classical Zero-Point Radiation}

\section{Zero-Point Radiation in Classical Physics}

Experimental measurements [9] have confirmed that Casimir forces can be described by random classical radiation[10] at temperature $T=0$. Thus any theory of classical thermal radiation must also included classical zero-point radiation as the limit when the temperature goes to absolute zero. We have seen above that, for non-zero temperature, a $\sigma_{l t U^{-1} \text {-scale }}$ transformation carries a situation at temperature $T$ into a situation at temperature $T / \sigma$. At the absolute zero of temperature, both $T$ and $T / \sigma$ are the same so that there can be no change under rescaling; accordingly we expect $\sigma_{l t U^{-1}}$-scale invariance for the random zeropoint radiation. Thus the vacuum should not have any finite-valued parameter associated

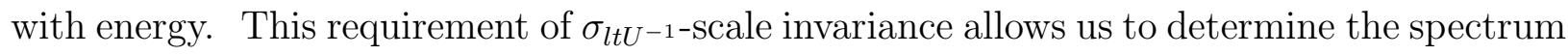
of classical zero-point radiation.

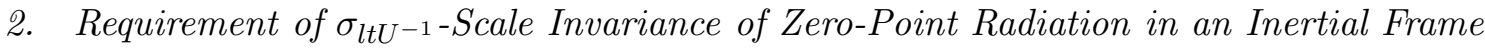

From the energy density equation (4) in two spacetime dimensions, we require that $\left(\partial_{x} \phi\right)^{2}$ have the dimensions of energy divided by length. Therefore $\phi^{2}$ has the dimensions of energy

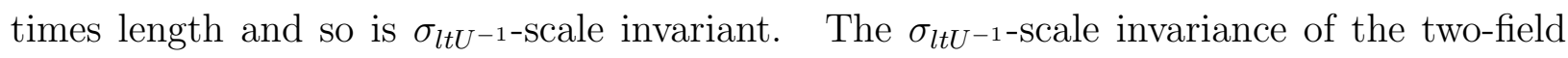
correlation function for zero-point radiation thus requires

$$
\left\langle\phi_{0}(c t, x) \phi_{0}\left(c t^{\prime}, x^{\prime}\right)\right\rangle=\left\langle\phi_{0}(c \sigma t, \sigma x) \phi_{0}\left(c \sigma t^{\prime}, \sigma x^{\prime}\right)\right\rangle
$$

From equation (26) in terms of the spectrum $f^{2}(|k|)$, this implies

$$
\begin{aligned}
& \int_{-\infty}^{\infty} d k f^{2}(|k|) \cos \left[k\left(x-x^{\prime}\right)-|k| c\left(t-t^{\prime}\right)\right] \\
& =\int_{-\infty}^{\infty} d k f^{2}(|k|) \cos \left[k\left(\sigma x-\sigma x^{\prime}\right)-|k| c\left(\sigma t-\sigma t^{\prime}\right)\right] \\
& =\int_{-\infty}^{\infty} d k^{\prime}\left(\sigma^{-1} f^{2}\left(\left|k^{\prime}\right| / \sigma\right)\right) \cos \left[k^{\prime}\left(x-x^{\prime}\right)-\left|k^{\prime}\right| c\left(t-t^{\prime}\right)\right]
\end{aligned}
$$

where we have made the change of variable $k^{\prime}=\sigma k, d k^{\prime}=\sigma k^{\prime},\left|k^{\prime}\right|=\sigma|k|$, for $\sigma>0$. Then the correlation function is $\sigma_{l t U^{-1}}$-scale invariant provided $\left(\sigma^{-1} f^{2}\left(\left|k^{\prime}\right| / \sigma\right)\right)=f^{2}\left(\left|k^{\prime}\right|\right)$, which means that

$$
f^{2}(|k|)=\text { const } /|k|
$$


Thus we have found the $\sigma_{l t U^{-1}}$-scale-invariant spectrum in an inertial frame in two spacetime dimensions. It turns out that this $\sigma_{l t U^{-1}}$-scale invariant spectrum is also Lorentz invariant.

\section{Lorentz Invariance of Zero-Point Radiation}

Since the field $\phi$ is a Lorentz scalar, the Lorentz invariance of the zero-point radiation corresponds to invariance of the correlation function under the transformation $c t \rightarrow \gamma c t-$ $\gamma \beta x, x \rightarrow \gamma x-\gamma \beta c t$; this corresponds to

$$
\left\langle\phi_{0}(c t, x) \phi_{0}\left(c t^{\prime}, x^{\prime}\right)\right\rangle=\left\langle\phi_{0}(\gamma c t-\gamma \beta x, \gamma x-\gamma \beta c t) \phi_{0}\left(\gamma c t^{\prime}-\gamma \beta x^{\prime}, \gamma x^{\prime}-\gamma \beta c t^{\prime}\right)\right\rangle
$$

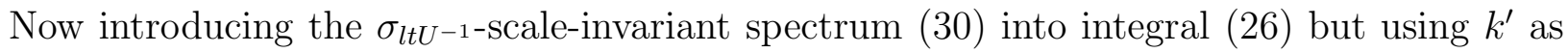
the (dummy) variable of integration, we have

$$
\begin{aligned}
& <\phi_{0}(\gamma c t-\gamma \beta x, \gamma x-\gamma \beta c t) \phi_{0}\left(\gamma c t^{\prime}-\gamma \beta x^{\prime}, \gamma x^{\prime}-\gamma \beta c t^{\prime}\right)>= \\
& =\int_{-\infty}^{\infty} d k^{\prime} \frac{\text { const }}{\left|k^{\prime}\right|} \cos \left[k^{\prime}\left\{\gamma\left(x-x^{\prime}\right)-\gamma \beta c\left(t-t^{\prime}\right)\right\}-\left|k^{\prime}\right|\left\{\gamma c\left(t-t^{\prime}\right)-\gamma \beta\left(x-x^{\prime}\right)\right\}\right] \\
& =\int_{-\infty}^{\infty} d k^{\prime} \frac{\text { const }}{\left|k^{\prime}\right|} \cos \left[\left(\gamma k^{\prime}+\gamma \beta\left|k^{\prime}\right|\right)\left(x-x^{\prime}\right)-\left(\gamma\left|k^{\prime}\right|+\gamma \beta k^{\prime}\right) c\left(t-t^{\prime}\right)\right] \\
& =\int_{-\infty}^{\infty} d k \frac{\text { const }}{|k|} \cos \left[k\left(x-x^{\prime}\right)-|k| c\left(t-t^{\prime}\right)\right] \\
& =<\phi_{0}(c t, x) \phi_{0}\left(c t^{\prime}, x^{\prime}\right)>
\end{aligned}
$$

where we have changed the variable of integration to $k=\gamma k^{\prime}+\gamma \beta\left|k^{\prime}\right|$ and have noted

$$
\frac{d k}{|k|}=\frac{\gamma\left(1+\beta k^{\prime} /\left|k^{\prime}\right|\right) d k^{\prime}}{\gamma\left|k^{\prime}\right|+\gamma \beta k^{\prime}}=\frac{d k^{\prime}}{\left|k^{\prime}\right|}
$$

Thus indeed the integral takes exactly the same form in both the primed and unprimed Lorentz frames, and the zero-point radiation spectrum is Lorentz invariant, taking the same form in every inertial frame.

\section{Multiplicative Constant Determining Zero-Point Radiation}

The constant involved in the classical zero-point radiation spectrum can be obtained from comparison with the experimentally observed Casimir forces associated with random classical electromagnetic fields. It turns out that this constant takes a numerical value which is immediately recognized as corresponding to the familiar Planck constant appearing in the 
energy per normal mode $U(k)=(1 / 2) \hbar c|k|=(1 / 2) \hbar \omega$. By analogy with the electromagnetic case, we choose the spectrum of random classical scalar radiation to also give the same energy per normal mode so that

$$
\frac{1}{2} \hbar c|k|=U(k)=\frac{1}{8 \pi} k^{2} f^{2}(|k|)
$$

The two-field correlation function then becomes

$$
\left\langle\phi_{0}(c t, x) \phi_{0}\left(c t^{\prime}, x^{\prime}\right)\right\rangle=\hbar c \int_{-\infty}^{\infty} \frac{d k}{|k|} \cos \left[k\left(x-x^{\prime}\right)-|k| c\left(t-t^{\prime}\right)\right]
$$

Thus Planck's constant enters this classical analysis. In keeping with nineteenth century ideas of physics, we could have expressed the unknown spectral value const $=|k| f^{2}(|k|)$ in terms of Stefan's constant which was measured in the 1870s; however, today Stefan's constant is far less familiar than Planck's constant. The connection between the two is given by $\sigma_{S}=\left(\pi^{2} k_{B}^{4}\right) /\left(15 \hbar^{3} c^{3}\right)$.[16]

Although zero-point energy is familiar to students from its appearance in quantum theory, most students are completely unaware of the possibility of classical zero-point radiation. Also, most students are unaware of the fact that an energy per normal mode given by a constant times the frequency of the mode corresponds to a radiation spectrum which is both

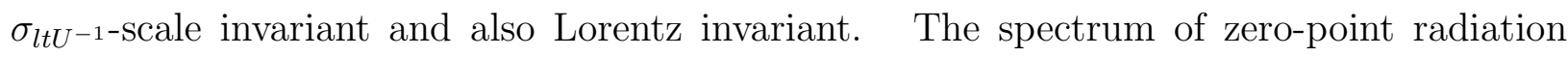
involves symmetries which are more fundamental than simply an adjustment value for the ground state of the quantum harmonic oscillator.

\section{Closed-Form Evaluation of the Two-Field Correlation Function in Zero-Point Radiation}

Since we know the exact form for the zero-point radiation spectrum, we can try to evaluate the integral in Eq. (35) for the two-field correlation function. Unfortunately the integrand is logarithmically divergent at small values of the wave number $k$. [17] Thus rather than evaluating the expression in Eq. (35), we will follow Fulling and Davies [18] and will evaluate the expressions involving the derivatives $\left\langle\phi_{0}(c t, x) \partial_{\mu^{\prime}} \phi_{0}\left(c t^{\prime}, x^{\prime}\right)\right\rangle$. Thus we have

$$
\left\langle\phi_{0}(c t, x) \partial_{c t^{\prime}} \phi_{0}\left(c t^{\prime}, x^{\prime}\right)\right\rangle=-\hbar c \int_{-\infty}^{\infty} \frac{d k}{|k|}|k| \sin \left[k\left(x-x^{\prime}\right)-|k| c\left(t-t^{\prime}\right)\right]
$$

and

$$
\left\langle\phi_{0}(c t, x) \partial_{x^{\prime}} \phi_{0}\left(c t^{\prime}, x^{\prime}\right)\right\rangle=\hbar c \int_{-\infty}^{\infty} \frac{d k}{|k|} k \sin \left[k\left(x-x^{\prime}\right)-|k| c\left(t-t^{\prime}\right)\right]
$$


Although the integrals in Eqs. (36) and (37) are now convergent at small wave number $k$, they still require a cut-off at large values of $|k|$ where the trigonometric functions oscillate increasingly rapidly. We will introduce an exponential cut off of the integrand. Thus the sort of integral which we must evaluate corresponds to the real or imaginary part of

$$
\begin{aligned}
\lim _{\Lambda \rightarrow 0_{+}} \int_{0}^{\infty} d k k^{n} \exp [(i b-\Lambda) k] & =\lim _{\Lambda \rightarrow 0_{+}} \frac{\partial^{n}}{\partial(i b-\Lambda)^{n}} \int_{0}^{\infty} d k \exp [(i b-\Lambda) k] \\
& =\lim _{\Lambda \rightarrow 0_{+}} \frac{\partial^{n}}{\partial(i b-\Lambda)^{n}}\left[\frac{\exp [(i b-\Lambda) k]}{(i b-\Lambda)}\right]_{k=0}^{k=\infty} \\
& =\frac{(-1)^{n+1}(n) !}{(i b)^{n+1}}
\end{aligned}
$$

Thus we obtain the non-zero singular Fourier sine and cosine transforms

$$
\int_{0}^{\infty} d k k^{2 m} \sin (b k)=\frac{(-1)^{2 m}(2 m) !}{b^{2 m+1}}
$$

and

$$
\int_{0}^{\infty} d k k^{2 m+1} \cos (b k)=\frac{(-1)^{2 m+1}(2 m+1) !}{b^{2 m+2}}
$$

Then it follows that for classical zero-point radiation, the correlation of Eq. (37) becomes

$$
\begin{aligned}
\left\langle\phi_{0}(c t, x) \partial_{x^{\prime}} \phi_{0}\left(c t^{\prime}, x^{\prime}\right)\right\rangle & =\hbar c \int_{-\infty}^{0} \frac{d k}{(-k)} k \sin \left[k\left(x-x^{\prime}\right)-(-k) c\left(t-t^{\prime}\right)\right] \\
& +\hbar c \int_{0}^{\infty} \frac{d k}{k} k \sin \left[k\left(x-x^{\prime}\right)-k c\left(t-t^{\prime}\right)\right] \\
& =\hbar c\left\{\frac{1}{\left(x-x^{\prime}\right)+c\left(t-t^{\prime}\right)}+\frac{1}{\left(x-x^{\prime}\right)-c\left(t-t^{\prime}\right)}\right\} \\
& =2 \hbar c \frac{\left(x-x^{\prime}\right)}{\left(x-x^{\prime}\right)^{2}-c^{2}\left(t-t^{\prime}\right)^{2}}
\end{aligned}
$$

and similarly Eq. (36) becomes

$$
\begin{aligned}
\left\langle\phi_{0}(c t, x) \partial_{c t^{\prime}} \phi_{0}\left(c t^{\prime}, x^{\prime}\right)\right\rangle & =\hbar c\left\{\frac{1}{\left(x-x^{\prime}\right)+c\left(t-t^{\prime}\right)}-\frac{1}{\left(x-x^{\prime}\right)-c\left(t-t^{\prime}\right)}\right\} \\
& =2 \hbar c \frac{-c\left(t-t^{\prime}\right)}{\left(x-x^{\prime}\right)^{2}-c^{2}\left(t-t^{\prime}\right)^{2}}
\end{aligned}
$$

We notice that, with the extra minus sign between the two correlations, they form a covariant (as opposed to contravariant) Lorentz vector, as would fit with the covariant partial derivative. We also notice that there is no parameter involving length, time, or energy which enters these expressions for the classical zero-point field in an inertial frame. 


\section{F. Thermal Radiation Spectrum Requirements in an Inertial Frame}

\section{1. $\sigma_{l t U^{-1}-S c a l i n g ~ B e h a v i o r}$ and Wien's Law}

When discussing the spectrum of random classical radiation in an inertial frame, we have so far considered only zero-point radiation. The spectrum of classical thermal radiation must be connected continuously with the spectrum of classical zero-point radiation as the temperature goes to zero. Thus the thermal spectrum $f^{2}(|k|, T)$ is a function of the wave number $k$ and the temperature $T$ such that $f^{2}(|k|, T) \rightarrow 4 \pi \hbar c /|k|$ as $T \rightarrow 0$. We also know that under an adiabatic change of the length of a box (or equivalently under a $\sigma_{l t U^{-1}}$ scale change), the thermal radiation changes so that the temperature $T \rightarrow T / \sigma$. Thus for thermal radiation at non-zero temperature, we go back to the two-point correlation function

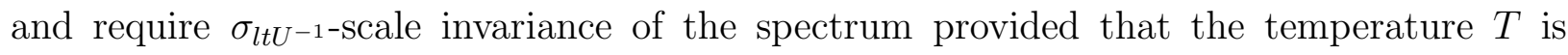
rescaled to $T / \sigma$, or $\left\langle\phi_{T / \sigma}(c \sigma t, \sigma x) \phi_{T / \sigma}\left(c \sigma t^{\prime}, \sigma x^{\prime}\right)\right\rangle=\left\langle\phi_{T}(c t, x) \phi_{T}\left(c t^{\prime}, x^{\prime}\right)\right\rangle$. The condition on the spectrum thus requires

$$
\begin{aligned}
& <\phi_{T / \sigma}(c \sigma t, \sigma x) \phi_{T / \sigma}\left(c \sigma t^{\prime}, \sigma x^{\prime}\right)>= \\
& =\frac{1}{4 \pi} \int_{-\infty}^{\infty} d k f^{2}(|k|, T / \sigma) \cos \left[k\left(\sigma x-\sigma x^{\prime}\right)-|k| c\left(\sigma t-\sigma t^{\prime}\right)\right] \\
& =\frac{1}{4 \pi} \int_{-\infty}^{\infty} d k f^{2}(|k|, T / \sigma) \cos \left[\sigma k\left(x-x^{\prime}\right)-|\sigma k| c\left(t-t^{\prime}\right)\right] \\
& =\frac{1}{4 \pi} \int_{-\infty}^{\infty}\left(d k^{\prime} / \sigma\right) f^{2}\left(\left|k^{\prime} / \sigma\right|, T / \sigma\right) \cos \left[k^{\prime}\left(x-x^{\prime}\right)-\left|k^{\prime}\right| c\left(t-t^{\prime}\right)\right] \\
& =\frac{1}{4 \pi} \int_{-\infty}^{\infty} d k^{\prime} f^{2}\left(\left|k^{\prime}\right|, T\right) \cos \left[k^{\prime}\left(x-x^{\prime}\right)-\left|k^{\prime}\right| c\left(t-t^{\prime}\right)\right] \\
& =<\phi_{T}(c t, x) \phi_{T}\left(c t^{\prime}, x^{\prime}\right)>
\end{aligned}
$$

Thus the required condition on the spectrum corresponds to

$$
\frac{1}{\sigma} f^{2}(|k| / \sigma, T / \sigma)=f^{2}(|k|, T)
$$

for all positive values of $\sigma$. The condition (44) has the general solution

$$
f^{2}(|k|, T)=\frac{1}{|k|} g\left(\frac{|k|}{T}\right)
$$

for an arbitrary function $g(|k| / T)$. This result corresponds to Wien's displacement law

for the thermal radiation spectrum. [4] Thus in an inertial frame, Wien's displacement law

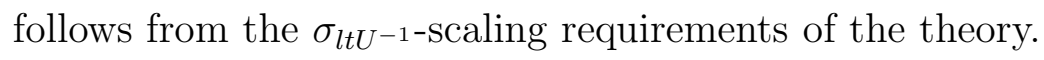


In the limit as the temperature goes to zero, we must recover the zero-point radiation spectrum. Also, we define the scale of temperature such that the energy of a normal mode in Eq. (21) is given by $k_{B} T$ at large temperature. Thus the limits on the function $g$ require from

$$
U(k)=(1 / 8 \pi) k^{2} f^{2}(|k|, T)=(1 / 8 \pi)|k| g^{2}(|k| / T)
$$

that

$$
g\left(\frac{|k|}{T}\right) \rightarrow 4 \pi \hbar c \text { for } k_{B} T<<\hbar c|k| \text { and } g\left(\frac{|k|}{T}\right) \rightarrow 8 \pi \frac{k_{B} T}{|k|} \text { for } T>>\hbar c|k|
$$

Also the function $g(|k| / T)$ must be a monotonically decreasing function of its argument so that the thermal radiation spectrum will be a monotonically increasing function of temperature. Thus determination of the spectrum of classical thermal radiation involves determining a single unknown function $g(|k| / T)$, and all thermal radiation spectra involve rescaled versions of this function. If we knew the spectrum for even one temperature $T>0$, then we could determine the spectrum for any other temperature $T^{\prime}=\sigma T$ by simply carrying out a

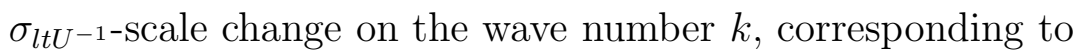

$$
\begin{aligned}
f^{2}\left(|k|, T^{\prime}\right) & =f^{2}(|k|, \sigma T)=(1 /|k|) g(|k| / \sigma T)= \\
(1 / \sigma) /(|k| / \sigma) g((|k| / \sigma) / T) & =(1 / \sigma) f^{2}(|k| / \sigma, T) .
\end{aligned}
$$

\section{Consistency with the Stefan-Boltzmann Relation}

From the functional form for thermal radiation corresponding to (45), it immediately follows that the thermal energy density $u_{T}$ follows the Stefan-Boltzmann relation obtained earlier from pure thermodynamics in Eq. (14). Thus the thermal energy density $u_{T}$ can be evaluated as the finite energy density above the divergent zero-point energy

$$
\begin{aligned}
u_{T}(x) & =\frac{1}{8 \pi}\left[\frac{1}{c^{2}}\left(\frac{\partial \phi_{T}}{\partial t}\right)^{2}+\left(\frac{\partial \phi_{T}}{\partial x}\right)^{2}\right]-\frac{1}{8 \pi}\left[\frac{1}{c^{2}}\left(\frac{\partial \phi_{0}}{\partial t}\right)^{2}+\left(\frac{\partial \phi_{0}}{\partial x}\right)^{2}\right] \\
& =\frac{1}{8 \pi}\left[\left(\frac{1}{c^{2}} \frac{\partial}{\partial t} \frac{\partial}{\partial t^{\prime}}+\frac{\partial}{\partial x} \frac{\partial}{\partial x^{\prime}}\right)\left\{<\phi_{T}(c t, x) \phi_{T}\left(c t^{\prime}, x^{\prime}\right)>-<\phi_{0}(c t, x) \phi_{0}\left(c t^{\prime}, x^{\prime}\right)>\right\}\right]_{t=t^{\prime}, x=x^{\prime}} \\
& =\frac{1}{8 \pi}\left[\frac{1}{4 \pi} \int_{-\infty}^{\infty} d k \frac{1}{|k|}[g(|v|)-4 \pi \hbar c] 2 k^{2} \cos \left[k^{\prime}\left(x-x^{\prime}\right)-\left|k^{\prime}\right| c\left(t-t^{\prime}\right)\right]\right]_{t=t^{\prime}, x=x^{\prime}} \\
& =\left(\frac{1}{4 \pi}\right)^{2} \int_{-\infty}^{\infty} d k|k|[g(|v|)-4 \pi \hbar c]=T^{2}\left[\left(\frac{1}{4 \pi}\right)^{2} \int_{-\infty}^{\infty} d v|v|[g(|v|)-4 \pi \hbar c]\right]=\sigma_{2 D} T^{2}
\end{aligned}
$$


where we have subtracted the integrands at given wave number $k$, and we have used the substitution $v=k / T$. The final integral gives the constant $\sigma_{2 D}$ which is independent of temperature $T$, and the result corresponds exactly to the thermodynamic result in Eq. (14).

\section{Correlation Lengths and Times for Thermal Radiation in an Inertial Frame}

Thermal radiation at non-zero temperature in a finite-length box involves a finite amount of radiation energy which is distributed over the radiation normal modes of the box. The thermal radiation is energy above the $\sigma_{l t U^{-1}}$-scale-invariant zero-point radiation. Since there are an infinite number of normal modes of ever-increasing frequency, the finite amount of thermal radiation must decrease as the frequency of the modes increases. We expect thermal radiation to be distributed in a smooth monotonic fashion with more thermal energy at lower frequencies. Since the zero-point energy per normal mode increases with frequency, there must be some normal mode where the thermal energy in the mode is comparable to the zero-point energy in the mode. The wavelength $\lambda_{T}$, frequency $\nu_{T}$, and energy $U\left(\nu_{T}, T\right)$ of this normal mode will provide a characteristic length, time, and energy related to the temperature for the thermal radiation distribution; thus we expect from Wien's displacement theorem $\lambda_{T}=$ const $/ T, \nu_{T}=c / \lambda_{T}=c T /$ const, and $U\left(\nu_{T}, T\right)=$ const $^{\prime} \times T$. Since all three parameters scale together in a thermal distribution, knowledge of any one parameter implies information about the other two. These parameters will be directly related to correlation lengths and correlation times for the thermal radiation. We emphasize that the $\sigma_{l t U^{-1-}}$ scale-invariant zero-point radiation has no such parameters in an inertial frame. Thus in an inertial frame, thermal radiation at non-zero-temperature $T$ is crucially different from zero-point radiation.

4. Correlation Function for a Single Spatial Point or Single Time for Thermal Radiation in an Inertial Frame

From the scaling information above in Eqs. (43) and (45), the two-point correlation function for the thermal radiation fields takes the form

$$
<\phi_{T}(c t, x) \phi_{T}\left(c t^{\prime}, x^{\prime}\right)>=\frac{1}{4 \pi} \int_{-\infty}^{\infty} \frac{d k}{|k|} g(|k| / T) \cos \left[k\left(x-x^{\prime}\right)-|k| c\left(t-t^{\prime}\right)\right]
$$


We would like to turn our information about the spectrum $g(|k| / T)$ into information about the time correlations at a single spatial point for use in our later analysis in a Rindler frame. In order to assure convergence of the integral at small values of $k$, we again consider the time derivative of Eq. (49) at $x=x^{\prime}$,

$$
\begin{aligned}
& <\phi_{T}(c t, x) \partial_{c t^{\prime}} \phi_{T}\left(c t^{\prime}, x\right)>=\frac{1}{4 \pi} \int_{-\infty}^{\infty} d k g(|k| / T) \sin \left[|k| c\left(t-t^{\prime}\right)\right] \\
& =T \frac{1}{4 \pi} \int_{0}^{\infty} d v[2 g(|v|)] \sin \left[|v| T c\left(t-t^{\prime}\right)\right]=T F\left[T c\left(t-t^{\prime}\right)\right]
\end{aligned}
$$

where we have introduced the change of integration variable $v=k / T$ for non-zero temperature $T$. Here the expression $F\left[T c\left(t-t^{\prime}\right)\right]$ is some unknown function of the temperature $T$ times the time difference $c\left(t-t^{\prime}\right)$. Because a rapidly varying sine function of $|k| T c\left(t-t^{\prime}\right)$ should cause cancellations between the positive and negative contributions in the integrand of Eq. (50), we would expect that at small time differences the correlation function should reflect the zero-point radiation spectrum $g(|k| / T) \rightarrow 4 \pi \hbar c$ at high frequencies, while at large time differences, the correlation function should reflect the low-frequency thermal radiation spectrum $g(|k| / T) \rightarrow 8 \pi k_{B} T /|k|$ as in Eq. (47). Also, at zero temperature, the correlation function should depend solely on the scale-invariant zero-point spectrum and therefore should depend upon the time difference but not on any parameter involving length, time, or energy. Thus we expect the limits

$$
T F\left[T c\left(t-t^{\prime}\right)\right] \rightarrow 2 \hbar c \frac{1}{c\left(t-t^{\prime}\right)} \quad \text { for } T c\left(t-t^{\prime}\right)<<1
$$

in connection with Eq. (42) for zero-point radiation, and we expect the limit

$$
T F\left[T c\left(t-t^{\prime}\right)\right] \rightarrow \text { const } \times T \quad \text { for } T c\left(t-t^{\prime}\right)>>1
$$

in connection with the Stefan-Boltzmann relation (14) and the energy density (49). Thus thermal radiation is a one-parameter family of radiation spectra determined by the spectral function $g(|k| / T)$ or by the correlation function $F\left[T c\left(t-t^{\prime}\right)\right]$.

We can also consider the correlation function at a fixed time $t=t^{\prime}$ at two different spatial points $x$ and $x^{\prime}$. The correlation with a non-zero derivative corresponds to the spatial derivative, which from Eq. (50) becomes

$$
\begin{aligned}
& <\phi_{T}(c t, x) \partial_{x^{\prime}} \phi_{T}\left(c t^{\prime}, x\right)>_{T}=\frac{1}{4 \pi} \int_{-\infty}^{\infty} \frac{d k}{|k|} g(|k| / T) k \sin \left[k\left(x-x^{\prime}\right)\right] \\
& =T \frac{1}{4 \pi} \int_{0}^{\infty} d v[2 g(|v|)] \sin \left[|v| T\left(x-x^{\prime}\right)\right]=T F\left[T\left(x-x^{\prime}\right)\right]
\end{aligned}
$$


We see that exactly the same unknown function $F$ is involved in the correlation function at a single time as is involved at a single spatial point. Thus in an inertial frame, we obtain the same information from the time correlations as from the spatial correlations of the scalar radiation field.

\section{PART II: THERMAL RADIATION IN A RINDLER FRAME}

\section{A. Introduction of a Rindler Frame}

\section{Analogy with Boltzmann's Use of Gravity}

Classical thermal radiation in an inertial frame seems to give no hint regarding the form of the spectrum at non-zero-temperature. In an inertial frame, zero-point radiation is

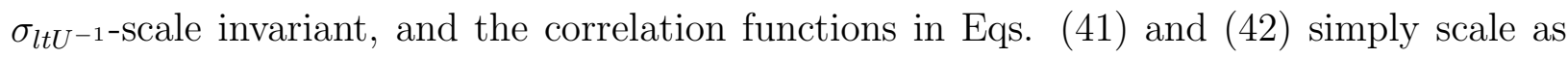

$\sigma^{-1}$ without giving any information about a functional form at non-zero-temperature. In order to get further information about the thermal spectrum, we do the same thing which Boltzmann did when dealing with the thermal velocity distribution for free particles in a box; he introduced a gravitational field and assumed that basic ideas of thermal equilibrium applied also in a gravitational field. Now by the equivalence principle, a gravitational field is locally equivalent to a coordinate frame undergoing constant acceleration relative to an inertial frame. Thus we will consider a one-dimensional box undergoing uniform acceleration relative to an inertial frame, and will assume that the basic ideas of thermodynamics hold in this accelerating frame.

\section{Rindler Coordinates}

Although the nonrelativistic mechanics of Boltzmann's analysis[11] allows a single constant acceleration throughout an accelerating box, this is not true in a relativistic analysis. The closest that we can come to a constant gravitational field is that provided by a Rindler coordinate system [5] [6] which is accelerating relative to an inertial frame with which it is instantaneously at rest at time $t=0$. If the time and space coordinates of the inertial frame are given by $(c t, x)$, then the connections with the time and space coordinates $(\eta, \xi)$ of the 
Rindler frame are

$$
\begin{aligned}
& c t=\xi \sinh \eta \\
& x=\xi \cosh \eta
\end{aligned}
$$

with $-\infty<\eta<\infty$, and $0<\xi$. Using the relationship $\cosh ^{2} \eta-\sinh ^{2} \eta=1$, it follows that a point with fixed spatial coordinate $\xi$ follows a trajectory

$$
x=\left(\xi^{2}+c^{2} t^{2}\right)^{1 / 2}
$$

in the inertial frame, and therefore undergoes a constant proper acceleration given by

$$
a_{\xi}=\left(\frac{d^{2} x}{d t^{2}}\right)_{t=0}=\frac{c^{2}}{\xi}
$$

Thus the proper acceleration $a_{\xi}$ of a coordinate point with fixed spatial coordinate $\xi$ becomes smaller as $\xi$ increases and becomes larger for small $\xi$. As $\xi \rightarrow 0$, the acceleration diverges, and the point where $\xi=0$ corresponds to an event horizon.

Now in thermal equilibrium in a gravitational field treated within a relativistic theory, the temperature must increase at points which are lower and must decrease at points which are higher according to the Tolman-Ehrenfest relation[19] $T\left(g_{00}\right)^{1 / 2}=$ const. Here in our two-dimensional spacetime, we have from Eqs. (1), (55), and (56)

$$
d s^{2}=c^{2} d t^{2}-d x^{2}=\xi^{2} d \eta^{2}-d \xi^{2}
$$

so that $g_{00}=\xi^{2}$, and therefore in the Rindler frame

$$
T \xi=\text { const }
$$

Thus, except at absolute zero, there is no single temperature which can be assigned to a box in a Rindler frame; rather, for $T>0$, the temperature at the bottom of the box must be larger than at the top of the box. Again under adiabatic compression, we expect the temperature in the box to increase. The temperature within a Rindler frame must

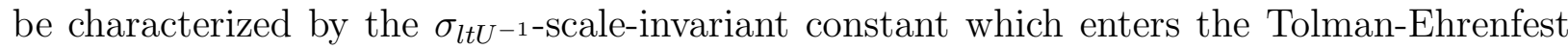
relation $(60)$.

\section{B. Radiation Normal Modes in a Rindler Frame}

At this point, we wish to consider the spectrum of random radiation as seen in the Rindler frame. First we obtain the radiation normal modes. The wave equation (3) in an 
inertial frame can be transformed to the wave equation in the Rindler frame by using the transformation equations (55), (56), together with the scalar behavior of the field $\phi$ under a coordinate transformation. The scalar field takes the same value in any coordinate frame. Thus the field $\varphi(\eta, \xi)$ in the Rindler frame is equal to the field $\phi(c t, x)$ in the inertial frame at the same spacetime point,

$$
\varphi(\eta, \xi)=\phi(c t, x)=\phi(\xi \sinh \eta, \xi \cosh \eta)
$$

Then using the usual rules for partial derivatives, we find that the wave equation (3) becomes in the Rindler frame

$$
\left(\frac{\partial^{2} \varphi}{\partial \xi^{2}}\right)+\frac{1}{\xi}\left(\frac{\partial \varphi}{\partial \xi}\right)-\frac{1}{\xi^{2}}\left(\frac{\partial^{2} \varphi}{\partial \eta^{2}}\right)=0
$$

The solutions of this Rindler wave equation take the form $H(\ln \xi \pm \eta)$ where $H$ is an arbitrary function. Thus whereas the general solution of the scalar wave equation (3) in an inertial frame is $\phi(c t, x)=h_{+}(x-c t)+h_{-}(x+c t)$ where $h_{+}$and $h_{-}$are arbitrary functions, the general solution in a Rindler frame is $\varphi(\eta, \xi)=H_{+}(\ln \xi-\eta)+H_{-}(\ln \xi+\eta)$ where $H_{+}$and $H_{-}$are arbitrary functions. The normal mode solutions of the wave equation in the Rindler frame for a box extending from $0<\xi=a$ to $\xi=b$ with Dirichlet boundary conditions can be obtained by separation of variables and can be written as

$$
\varphi_{n}(\eta, \xi)=\mathcal{F}_{n}\left(\frac{2}{\ln (b / a)}\right)^{1 / 2} \sin \left[\frac{n \pi}{\ln (b / a)} \ln \left(\frac{\xi}{a}\right)\right] \cos \left[\frac{n \pi}{\ln (b / a)} \eta+\theta_{n}\right], \quad n=1,2,3 \ldots
$$

where the spatial functions

$$
\psi_{n}(\eta, \xi)=\left(\frac{2}{\ln (b / a)}\right)^{1 / 2} \sin \left[\frac{n \pi}{\ln (b / a)} \ln \left(\frac{\xi}{a}\right)\right]
$$

arise from a Sturm-Liouville system[20] and so form a complete orthonormal set with weight $1 / \xi$ on the interval $a<\xi<b$

$$
\begin{aligned}
\int_{a}^{b} \frac{d \xi}{\xi} \psi_{n}(\xi) \psi_{m}(\xi) & = \\
& =\int_{a}^{b} \frac{d \xi}{\xi} \frac{2}{\ln (b / a)} \sin \left[\frac{n \pi}{\ln (b / a)} \ln \left(\frac{\xi}{a}\right)\right] \sin \left[\frac{m \pi}{\ln (b / a)} \ln \left(\frac{\xi}{a}\right)\right] \\
& =\int_{v=0}^{v=\pi} \frac{\ln (b / a)}{\pi} d v \frac{2}{\ln (b / a)} \sin n v \sin m v \\
& =\delta_{n m}
\end{aligned}
$$


where we have used the substitution $v=[\pi \ln (\xi / a)] / \ln (b / a)$ in evaluating the integral. For a radiation normal mode, the Rindler time parameter $\eta$ agrees with all local clocks when adjusted by $\xi$; thus the time $t=\xi \eta$ gives the proper time of a clock located at fixed Rindler spatial coordinate $\xi$.

For time-stationary random radiation, the field $\varphi(\eta, \xi)$ can be written as a sum over the normal modes $\varphi_{n}(\eta, \xi)$ in (62) with random phases $\theta_{n}$ distributed randomly over the interval $[0,2 \pi)$ and distributed independently for each value of $n$. Then the two-field correlation function is obtained in analogy with Eq. (22)-(24). For a large box, we can go through the same sort of analysis as is given in Eq. (24)-(26) to obtain a set of normal modes dependent upon a continuous wave number $\kappa=n \pi / \ln (b / a)$ and can accommodate both cosine functions by including the integral over negative values of $\kappa$, exactly as in Eq. (26). Here for the Rindler frame, we obtain the expression analogous to Eq. (26) in an inertial frame,

$$
\left\langle\varphi(\eta, \xi) \varphi\left(\eta^{\prime}, \xi^{\prime}\right)\right\rangle=\frac{1}{4 \pi} \int_{-\infty}^{\infty} d \kappa \mathcal{F}^{2}(|\kappa|) \cos \left[\kappa\left(\ln \xi-\ln \xi^{\prime}\right)-|\kappa|\left(\eta-\eta^{\prime}\right)\right]
$$

\section{C. $\sigma_{l t U^{-1}-\text { Scale Change in a Rindler Frame }}$}

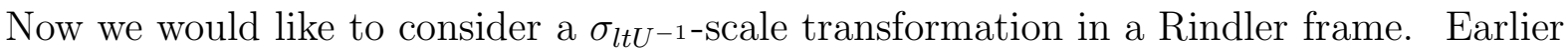

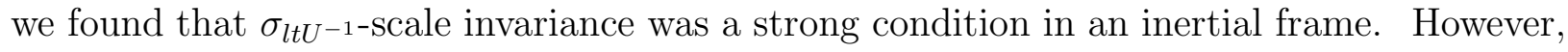
for a Rindler frame, we notice from the coordinate transformation equations $c t=\xi \sinh \eta$

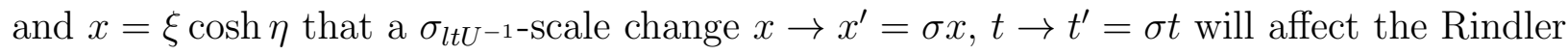
spatial coordinate $\xi$ but not the Rindler time coordinate $\eta$; thus we have $\xi \rightarrow \xi^{\prime}=\sigma \xi$, but $\eta \rightarrow \eta^{\prime}=\eta$, and $d s^{2} \rightarrow d s^{2}=\sigma^{2} d s^{2}=\xi^{\prime 2} d \eta^{\prime 2}-d \xi^{\prime 2}$. It follows from Eq. (65) and

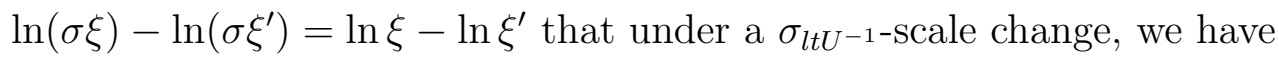

$$
\begin{aligned}
\left\langle\varphi(\eta, \sigma \xi) \varphi\left(\eta^{\prime}, \sigma \xi^{\prime}\right)\right\rangle & =\frac{1}{4 \pi} \int_{-\infty}^{\infty} d \kappa \mathcal{F}^{2}(|\kappa|) \cos \left[\kappa\left\{\ln (\xi)-\ln \left(\xi^{\prime}\right)\right\}-|\kappa|\left(\eta-\eta^{\prime}\right)\right] \\
& =\left\langle\varphi(\eta, \xi) \varphi\left(\eta^{\prime}, \xi^{\prime}\right)\right\rangle
\end{aligned}
$$

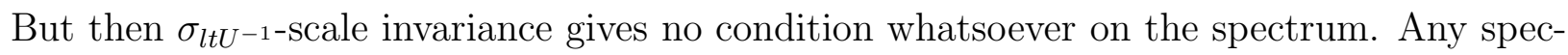
trum which is time-stationary in a Rindler frame is $\sigma_{l t U^{-1}-\text { scale invariant. The scaling }}$ operation simply carries the spatial points to new locations within the Rindler frame without imposing any restriction on the time-stationary spectrum of random radiation. This 
result is completely different from the requirements of $\sigma_{l t U^{-1}-\text { scale invariance in an inertial }}$ frame.

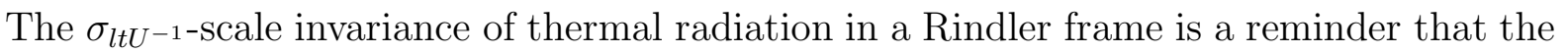
temperature of thermal radiation in a large box in a Rindler frame has no unique value but rather changes continuously throughout the box. Thermal radiation in a Rindler frame is

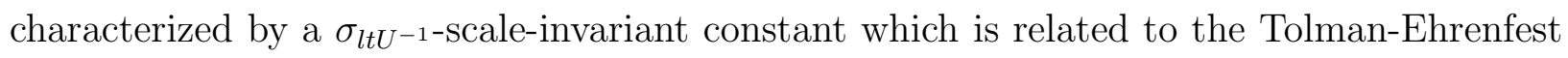

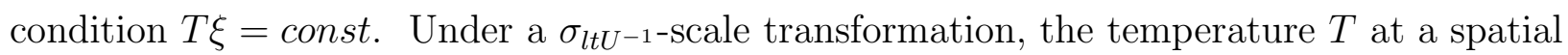
point $\xi$ is mapped so that $T \rightarrow T^{\prime}=T / \sigma$ while the spatial coordinate $\xi$ is mapped so that $\xi \rightarrow \xi^{\prime}=\sigma \xi$, leaving unchanged the relationship $T^{\prime} \xi^{\prime}=(T / \sigma)(\sigma \xi)=$ const

\section{Zero-Point Radiation in a Rindler Frame}

Classical physics deals with tensor quantities, and the zero-point radiation which is present in an inertial frame must also be present in a Rindler frame which is accelerating relative to an inertial frame. Furthermore, we expect that the thermal radiation which is present in a Rindler frame must fit with the zero-point radiation in the Rindler frame. We can obtain the form taken by the random zero-point radiation in the Rindler frame by noting that the field $\phi$ is a scalar under coordinate transformation so that the field $\varphi(\eta, \xi)$ in the Rindler frame is equal to the field $\phi(c t, x)$ in the inertial frame at the same spacetime point, as given in Eq. (61). Therefore we have from Eqs. (41) and (42) and from the transformations of Eqs. (55) and (56)

$$
\begin{aligned}
& <\varphi_{0}(\eta, \xi) \frac{\partial}{\partial \eta^{\prime}} \varphi_{0}\left(\eta^{\prime}, \xi^{\prime}\right)>= \\
& =<\phi(c t, x) \frac{\partial}{\partial c t^{\prime}} \phi\left(c t^{\prime}, x^{\prime}\right)>\frac{\partial c t^{\prime}}{\partial \eta^{\prime}}+<\phi(c t, x) \frac{\partial}{\partial x^{\prime}} \phi\left(c t^{\prime}, x^{\prime}\right)>\frac{\partial x^{\prime}}{\partial \eta^{\prime}} \\
& =2 \hbar c \frac{-c\left(t-t^{\prime}\right)}{\left(x-x^{\prime}\right)^{2}-c^{2}\left(t-t^{\prime}\right)^{2}} \xi^{\prime} \cosh \eta^{\prime}+2 \hbar c \frac{\left(x-x^{\prime}\right)}{\left(x-x^{\prime}\right)^{2}-c^{2}\left(t-t^{\prime}\right)^{2}} \xi^{\prime} \sinh \eta^{\prime} \\
& =2 \hbar c \frac{\xi \xi^{\prime} \sinh \left(\eta-\eta^{\prime}\right)}{\xi^{2}+\xi^{\prime 2}-2 \xi \xi^{\prime} \cosh (\eta-\eta)}
\end{aligned}
$$


and similarly

$$
\begin{aligned}
& <\varphi_{0}(\eta, \xi) \frac{\partial}{\partial \xi^{\prime}} \varphi_{0}\left(\eta^{\prime}, \xi^{\prime}\right)>= \\
& =<\phi(c t, x) \frac{\partial}{\partial c t^{\prime}} \phi\left(c t^{\prime}, x^{\prime}\right)>\frac{\partial c t^{\prime}}{\partial \xi^{\prime}}+<\phi(c t, x) \frac{\partial}{\partial x^{\prime}} \phi\left(c t^{\prime}, x^{\prime}\right)>\frac{\partial x^{\prime}}{\partial \xi^{\prime}} \\
& =2 \hbar c \frac{-c\left(t-t^{\prime}\right)}{\left(x-x^{\prime}\right)^{2}-c^{2}\left(t-t^{\prime}\right)^{2}} \sinh \eta^{\prime}+2 \hbar c \frac{\left(x-x^{\prime}\right)}{\left(x-x^{\prime}\right)^{2}-c^{2}\left(t-t^{\prime}\right)^{2}} \cosh \eta^{\prime} \\
& =2 \hbar c \frac{\xi \cosh \left(\eta-\eta^{\prime}\right)-\xi^{\prime}}{\xi^{2}+\xi^{\prime 2}-2 \xi \xi^{\prime} \cosh (\eta-\eta)}
\end{aligned}
$$

We notice immediately that these expressions involve only the time difference $\eta-\eta^{\prime}$ in the Rindler frame, and not the specific times $\eta$ and $\eta^{\prime}$. Thus the zero-point radiation is a time-stationary distribution in a Rindler frame. Indeed the $\sigma_{l t U^{-1-s c a l e-i n v a r i a n t} \text { and }}$ Lorentz-invariant zero-point radiation is the only spectrum of random classical radiation which is time-stationary in both all inertial frames and all Rindler frames.

At a single time $\eta=\eta^{\prime}$, but at two different spatial points $\xi$ and $\xi^{\prime}$, we find from Eqs. (68) and (69) that $\left\langle\varphi_{0}(\eta, \xi) \frac{\partial}{\partial \eta^{\prime}} \varphi_{0}\left(\eta, \xi^{\prime}\right)\right\rangle=0$, while

$$
\left\langle\varphi_{0}(\eta, \xi) \frac{\partial}{\partial \xi^{\prime}} \varphi_{0}\left(\eta, \xi^{\prime}\right)\right\rangle=\frac{2 \hbar c}{\xi-\xi^{\prime}}
$$

However, this equation (70) is exactly the same correlation function as appears in Eq. (41) for zero-point radiation in an inertial frame when $t=t^{\prime}$. Indeed, a single time in a Rindler frame is also a single time in the inertial frame with which the Rindler frame is momentarily at rest, so that the correlation function involving different spatial points given in Eq. (70) can be interpreted as the correlation function at a single time in either the Rindler frame or the associated inertial frame. Thus this correlation function in the Rindler frame gives us no new information beyond that of an inertial frame.

At a single Rindler spatial coordinate $\xi$, but at two different times $\eta$ and $\eta^{\prime}$, the correlation functions (68) and (69) become

$$
\begin{aligned}
\left\langle\varphi_{0}(\eta, \xi) \frac{\partial}{\partial \eta^{\prime}} \varphi_{0}\left(\eta^{\prime}, \xi^{\prime}\right)\right\rangle_{\xi^{\prime}=\xi} & =2 \hbar c \frac{2 \sinh \left[\left(\eta-\eta^{\prime}\right) / 2\right] \cosh \left[\left(\eta-\eta^{\prime}\right) / 2\right]}{4 \sinh ^{2}\left[\left(\eta-\eta^{\prime}\right) / 2\right]} \\
& =\hbar c \operatorname{coth}\left(\frac{\eta-\eta^{\prime}}{2}\right)
\end{aligned}
$$

and

$$
\left\langle\varphi_{0}(\eta, \xi) \frac{\partial}{\partial \xi^{\prime}} \varphi_{0}\left(\eta^{\prime}, \xi^{\prime}\right)\right\rangle_{\xi^{\prime}=\xi}=-\frac{\hbar c}{\xi}
$$

These expressions are quite different from the analogous expressions for zero-point radiation in an inertial frame found from (41) and (42) when $x=x^{\prime}$. 


\section{E. From Zero-Point Radiation to Thermal Radiation in a Rindler Frame}

\section{Scaling Parameter for Thermal Radiation in a Rindler Frame}

We notice that the zero-point expression $\left\langle\varphi_{0}(\eta, \xi) \partial_{\eta^{\prime}} \varphi_{0}\left(\eta^{\prime}, \xi^{\prime}\right)\right\rangle_{\xi^{\prime}=\xi}$ given in Eq. (71) has no dependence upon the spatial coordinate $\xi$, but does have a dependence upon the Rindler time difference $\eta-\eta^{\prime}$. We expect the thermal correlation function $<\varphi_{\alpha}(\eta, \xi) \partial_{\eta^{\prime}} \varphi_{\alpha}\left(\eta^{\prime}, \xi^{\prime}\right)>_{\xi^{\prime}=\xi}$

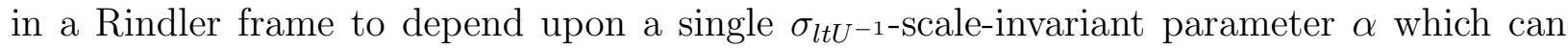
take on real values corresponding to the relationship $T \xi=$ const. The time correlation at a single spatial coordinate at non-zero temperature, when expressed in terms of the Rindler time parameter $\eta$, should be the same at any point of the Rindler frame with no dependence upon the position coordinate $\xi$. Furthermore, the time correlation function must involve the thermal parameter $\alpha$ as a factor in connection with the time difference $\eta-\eta^{\prime}$. From our considerations of the time correlation for thermal radiation in an inertial frame appearing

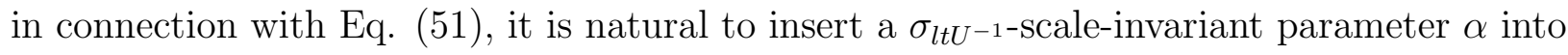
the Rindler time correlation (71) as

$$
\left\langle\varphi_{\alpha}(\eta, \xi) \frac{\partial}{\partial \eta^{\prime}} \varphi_{\alpha}\left(\eta^{\prime}, \xi^{\prime}\right)\right\rangle_{\xi^{\prime}=\xi}=\alpha \hbar c \operatorname{coth}\left(\alpha \frac{\eta-\eta^{\prime}}{2}\right)
$$

When $\alpha=1$, the situation corresponds to zero-point radiation in the Rindler frame, as given in Eq. (71). When $\alpha>1$, the situation corresponds to thermal radiation at a temperature greater than absolute zero. We notice that the thermal correlation given in Eq. (73) is a monotonically increasing function of $\alpha$ which has the asymptotic limits required for thermal radiation in Eqs. (52) and (53).

\section{Thermal Radiation Spectrum in a Rindler Frame}

The spectrum $\mathcal{F}_{\alpha}^{2}(|\kappa|)$ for thermal radiation in a Rindler frame can be obtained from the correlation-function expressions given in Eqs. (66) and (73) which require

$$
\begin{aligned}
\left\langle\varphi_{\alpha}(\eta, \xi) \frac{\partial}{\partial \eta^{\prime}} \varphi_{\alpha}\left(\eta^{\prime}, \xi^{\prime}\right)\right\rangle_{\xi^{\prime}=\xi} & =\frac{1}{4 \pi} \int_{-\infty}^{\infty} d \kappa \mathcal{F}_{\alpha}^{2}(|\kappa|)|\kappa| \sin \left[|\kappa|\left(\eta-\eta^{\prime}\right)\right] \\
& =\alpha \hbar c \operatorname{coth}\left(\alpha \frac{\eta-\eta^{\prime}}{2}\right)
\end{aligned}
$$

We can take the inverse Fourier sine transform of Eq. (74). The singular Fourier sine transform can be broken into a singular piece involving the same integral as in Eq. (39) and 
also a convergent piece which can be found in a standard table of integrals.[21] Thus we have

$$
\begin{aligned}
\mathcal{F}_{\alpha}^{2}(|\kappa|)|\kappa| & =4 \int_{0}^{\infty} d \eta\left[\alpha \hbar c \operatorname{coth}\left(\alpha \frac{\eta}{2}\right)\right] \sin (|\kappa| \eta) \\
& =4 \int_{0}^{\infty} d \eta(\alpha \hbar c)\left[1+\frac{2}{\exp (\alpha \eta)-1}\right] \sin (|\kappa| \eta) \\
& =4(\hbar c)\left[\frac{\alpha}{|\kappa|}+2\left\{\frac{\pi}{2} \operatorname{coth}\left(\frac{|\kappa| \pi}{\alpha}\right)-\frac{\alpha}{2|\kappa|}\right\}\right] \\
& =4 \pi \hbar c \operatorname{coth}\left(\frac{|\kappa| \pi}{\alpha}\right)
\end{aligned}
$$

as the spectrum for thermal radiation in a Rindler frame.

\section{The Vacuum State in a Rindler Frame}

In equation (75), the normal-mode spectrum for non-zero thermal radiation involves $\alpha>1$, whereas the spectrum corresponding to zero-point radiation corresponds to $\alpha=1$. Thus zero-point radiation as seen in a Rindler frame has the same basic spectral form as does thermal radiation. This situation is quite different from that of an inertial frame where zero-point radiation involves the power-law spectrum of Eq. (34) $f^{2}(|k|)=4 \pi \hbar c /|k|$ and gives no hint of the spectral form for non-zero temperature. It seems to come as a shock that in a Rindler frame the vacuum state does not have a spectrum of random radiation involving a power of the Rindler frequency $|\kappa|$ but rather has a more complicated functional form reflecting the non-inertial character of the coordinate frame. An analogous situation

arises for the electric field lines of a point charge; the electric field lines point radially away from the charge in all inertial frames, and yet change character so as to "droop" when undergoing the acceleration of a Rindler frame.

\section{F. Transferring Results from a Rindler Frame to an Inertial Frame}

Once we have the spectrum for classical thermal radiation in a Rindler frame, we can transfer this information back to an inertial frame by treating a box of thermal radiation which is far from the event horizon of the Rindler frame. The acceleration of a fixed spatial coordinate $\xi$ is given by $a_{\xi}=c^{2} / \xi$ and so becomes smaller as the distance $\xi$ from the event horizon becomes larger. The limit $a_{\xi} \rightarrow 0$ corresponds to an inertial frame. 
We imagine our box of thermal radiation as extending from a lower edge $\xi=a$ to an upper edge $\xi=b$, and we introduce a spatial coordinate $\bar{x}=\xi-a$, the time $c \bar{t}=a \eta$, and the length of the box $l=b-a$. Then the normal mode in the Rindler frame given in Eq. (63) can be rewritten for box length $l<<a$ as

$$
\begin{aligned}
\varphi_{n}(\eta, \xi) & =\phi(c \bar{t}, \bar{x})= \\
& =\left(\frac{2}{\ln [(a+l) / a]}\right)^{1 / 2} \sin \left[\frac{n \pi}{\ln [(a+l) / a]} \ln \left(\frac{a+\bar{x}}{a}\right)\right] \cos \left[\frac{n \pi}{\ln [(a+l) / a]} \frac{\bar{t}}{a}+\theta_{n}\right] \\
& \approx\left(\frac{2 a}{l}\right)^{1 / 2} \sin \left[\frac{n \pi a}{l} \frac{\bar{x}}{a}\right] \cos \left[\frac{n \pi a}{l} \frac{\bar{t}}{a}+\theta_{n}\right] \\
& =a^{1 / 2}\left(\frac{2}{l}\right)^{1 / 2} \sin \left[\frac{n \pi \bar{x}}{l}\right] \cos \left[\frac{n \pi \bar{t}}{l}+\theta_{n}\right]
\end{aligned}
$$

where we have used the approximation $\ln (1+l / a) \approx l / a$ which is valid for large $a, a>>$. The over-all factor of $a^{1 / 2}$ is compensated by the factor of $1 / \xi=1 /(a+\bar{x}) \approx 1 / a$ which arises in the normalization of the normal modes as seen in Eq. (65). Thus as the box of radiation considered is further and further away from the event horizon, the radiation normal modes go over to the normal modes (18) for a box in an inertial frame.

Next we consider the correlation function (73) in the limit of great distance from the event horizon; this function becomes

$$
\begin{aligned}
& <\varphi_{\alpha}(\eta, \xi) \frac{\partial}{\partial \eta^{\prime}} \varphi_{\alpha}\left(\eta^{\prime}, \xi^{\prime}\right)>_{\xi^{\prime}=\xi}=<\phi_{\alpha}(c \bar{t}, \bar{x}) a \frac{\partial}{\partial(c \bar{t})} \phi_{\alpha}\left(c \bar{t}^{\prime}, \bar{x}^{\prime}\right)>_{\bar{x}=\bar{x}^{\prime}} \\
& =\alpha \hbar c \operatorname{coth}\left(\alpha \frac{\eta-\eta^{\prime}}{2}\right)=\alpha \hbar c \operatorname{coth}\left(\alpha \frac{c\left(\bar{t}-\bar{t}^{\prime}\right)}{2 a}\right)
\end{aligned}
$$

Dividing Eq. (77) through by the factor $a$ (corresponding to the distance of the lower end of the box from the event horizon), we have

$$
\left\langle\phi_{\alpha}(c \bar{t}, \bar{x}) \frac{\partial}{\partial(c \bar{t})} \phi_{\alpha}\left(c \bar{t}^{\prime}, \bar{x}^{\prime}\right)\right\rangle_{\bar{x}=\bar{x}^{\prime}}=\frac{\alpha}{a} \hbar c \operatorname{coth}\left(\frac{\alpha}{a} \frac{c\left(\bar{t}-\bar{t}^{\prime}\right)}{2}\right)
$$

Now if, for fixed Rindler thermal parameter $\alpha$, we take the limit as the distance $a$ of the lower end of the box becomes increasingly far from the event horizon, then we find from the power series expansion $\operatorname{coth} z=1 / z+z / 3-\ldots$ that

$$
\begin{aligned}
& <\phi_{\alpha}(c \bar{t}, \bar{x}) \frac{\partial}{\partial(c \bar{t})} \phi_{\alpha}\left(c \bar{t}^{\prime}, \bar{x}^{\prime}\right)>_{\bar{x}=\bar{x}^{\prime}}=\frac{\alpha}{a} \hbar c\left(\frac{a}{\alpha} \frac{2}{c\left(\bar{t}-\bar{t}^{\prime}\right)}+\frac{1}{3} \frac{\alpha}{a} c \frac{\left(\bar{t}-\bar{t}^{\prime}\right)}{2}+\ldots\right) \\
& =2 \hbar c \frac{1}{c\left(\bar{t}-\bar{t}^{\prime}\right)} \text { for the limit } a \rightarrow \infty
\end{aligned}
$$


Thus for fixed Rindler thermal parameter $\alpha$, we simply recover Eq. (52) (the zero-point radiation spectrum in an inertial frame) in the limit $a \rightarrow \infty$ corresponding to being far from the event horizon. However, from the Tolman-Ehrenfest relation (60), the temperature $T$ in the box in the Rindler frame falls off with distance $a$ as $T=$ const/a. Thus as we consider a box whose lower edge $a$ is increasingly far from the event horizon, the temperature indeed becomes ever smaller and goes to zero in the limit of infinite distance.

If we want to carry a box of thermal radiation at non-zero temperature $T$ back to an inertial frame, then we must keep on increasing the Rindler thermal parameter $\alpha$ as the box considered is moved ever further from the event horizon. If we compare the expression (77) for the correlation function in a Rindler frame but very far from the event horizon with the correlation function (51) for thermal radiation in an inertial frame, we see that the ratio $\alpha / a$ must be proportional to the temperature $T$ at the lower end of the box, which temperature we wish to keep fixed. Accordingly, the unknown function $T F\left[T c\left(t-t^{\prime}\right)\right]$ in the inertial frame arising from the correlation function in Eq. (51) is given by

$$
<\phi_{T}(c \bar{t}, \bar{x}) \partial_{c \bar{t}} \phi_{T}\left(c \bar{t}^{\prime}, \bar{x}^{\prime}\right)>_{\bar{x}=\bar{x}^{\prime}}=T F\left[T c\left(t-t^{\prime}\right)\right]=\frac{\alpha}{a} \hbar c \operatorname{coth}\left(\frac{\alpha}{a} \frac{c\left(\bar{t}-\bar{t}^{\prime}\right)}{2}\right)
$$

where there is some constant of proportionality between the temperature $T$ and the ratio $\alpha / a$.

The spectral function for thermal radiation in an inertial frame $f^{2}(|k|, T)|k|=g(|k| / T)$ can be found from Eq. (80) by the same inverse Fourier sine transform as was evaluated in Eq. (75), and so we finally obtain the spectrum for classical scalar thermal radiation in an inertial frame

$$
\begin{aligned}
f^{2}(|k|, T)|k| & =g(|k| / T)=4 \int_{0}^{\infty} d t c\left[\frac{\alpha}{a} \hbar c \operatorname{coth}\left(\frac{\alpha}{a} \frac{c t}{2}\right)\right] \sin (|k| c t) \\
& =4 \pi \hbar c \operatorname{coth}\left(\frac{|k| \pi}{\alpha / a}\right)
\end{aligned}
$$

The proportionality constant between $T$ and $\alpha / a$ can be fixed by the limits of Eqs. (46) and (47) requiring that the energy $U(k)$ of a normal mode become $k_{B} T$ for fixed wave number $k$ and high temperature $T$. Then we find

$$
f^{2}(|k|, T)|k|=g(|k| / T)=4 \pi \hbar c \operatorname{coth}\left(\frac{\hbar c|k|}{2 k_{B} T}\right)
$$

and an energy spectrum corresponding to an energy per normal mode at wave number $k$ 
with $\omega=c|k|$,

$$
\begin{aligned}
U(k) & =\frac{1}{8 \pi} f^{2}(|k|, T) k^{2}=\frac{1}{2} \hbar c|k| \operatorname{coth}\left(\frac{\hbar c|k|}{2 k_{B} T}\right) \\
& =\frac{1}{2} \hbar \omega \operatorname{coth}\left(\frac{\hbar \omega}{2 k_{B} T}\right)=\frac{1}{2} \hbar \omega+\frac{1}{\exp \left(\hbar \omega / k_{B} T\right)-1}
\end{aligned}
$$

This result is just the Planck spectrum of thermal radiation including zero-point radiation. Thus we find that by considering the box of thermal radiation ever farther from the event horizon while keeping the temperature inside the box fixed, the coordinates become ever closer to inertial coordinates, the normal modes go over to inertial-frame normal modes, and the spectrum becomes the thermal radiation spectrum in an inertial frame. Thus we have successfully obtained the Planck spectrum of thermal radiation from the use of zero-point radiation and the information of a relativistic Rindler frame.

What is thermal radiation? Apparently thermal radiation is time-stationary radiation in an inertial frame which follows the same basic spectral form as Lorentz-invariant zero-point radiation takes in a Rindler frame.

\section{DISCUSSION}

All the textbooks[2] of physics claim that classical physics is incapable of obtaining the Planck spectrum of thermal radiation and that one must use the quantum statical mechanics of photons in order to account for thermal radiation. However, the usual classical analysis applies nonrelativistic classical statistical mechanics[22] to the radiation modes of the relativistic radiation field or else treats the scattering of the relativistic radiation field by nonrelativistic mechanical scatterers. 23] These calculations involve inconsistent mixtures of Galilean-invariant and Lorentz-invariant physics. The present analysis obtains the Planck spectrum within a consistent relativistic analysis. Again it is suggested that only a fully relativistic treatment of relativistic radiation is consistent with classical radiation equilibrium. This point of view agrees with the scattering result reported for electromagnetic radiation that classical zero-point radiation remains zero-point radiation under scattering by a relativistic charged particle in a Coulomb potential. [24] 


\section{ACKNOWLEDGEMENT}

The present analysis is related to work in quantum field theory [25] [26] which deals with the "thermal effects of acceleration." [27] It was first within the context of the quantum field theory that there arose a connection between thermal radiation and acceleration. Work from the quantum calculations has provided hints for the classical analysis presented here.

[1] See for example, P. M. Morse, Thermal Physics 2nd ed. (Benjamin/Cummins, Reading, MA 1981).

[2] See for example, R. Eisberg and R. Resnick, Quantum Physics of Atoms, Molecules, Solids, Nuclei, and Particles 2nd ed. (Wiley, New York 1985).

[3] See for example, ref. 1, pp. 78-79.

[4] See for example, B. H. Lavenda, Statistical Physics: A Probabilistic Approach (Wiley, New York 1991), pp. 67-70.

[5] See for example, B. F. Schutz, A First Course in General Relativity (Cambridge U. Press 1986), p. 150.

[6] See for example, W. Rindler, Essential Relativity: Special, General, and Cosmological 2nd ed. (Springer-Verlag, New York 1977), pp. 49-51.

[7] In two spacetime dimensions, the analysis involves only trigonometric and exponential functions. In four spacetime dimensions, the Rindler-frame normal modes involve modified Bessel functions of imaginary argument.

[8] See for example, ref. 4, p. 63.

[9] M. J. Sparnaay, "Measurement of the attractive forces between flat plates," Physica (Amsterdam) 24, 751-764 (1958); S. K. Lamoreaux, "Demonstration of the Casimir force in the 0.6 to $6 \mu \mathrm{m}$ range," Phys. Rev. Lett. 78, 5-8 (1997): 81, 5475-5476 (1998); U. Mohideen, "Precision measurement of the Casimir force from 0.1 to $0.9 \mu \mathrm{m}$," ibid. 81, 4549-4552 (1998); H. B. Chan, V. A. Aksyuk, R. N. Kleinman, D. J. Bishop, and F. Capasso, "Quantum mechanical actuation of microelectromechanical systems by the Casimir force," Science 291, 1941-1944 (2001): G. Bressi, G. Caarugno, R. Onofrio, and G. Ruoso, "Measurement of the Casimir force between parallel metallic surfaces," Phys. Rev. Lett. 88, 041804(4) (2002). 
[10] The original calculation was made in terms of the zero-point energy of quantum field theory by H. B. G. Casimir, "On the attraction between two perfectly conducting plates," Proc. Ned. Akad. Wetenschap. 51, 793-795 (1948). However, the same results appear in classical electrodynamics which includes classical electromagnetic zero-point radiation. See for example, T. H. Boyer, "Random electrodynamics: The theory of classical electrodynamics with classical electromagnetic zero-point radiation," Phys. Rev. 11, 790-808 (1976).

[11] Boltzmann's derivation is discussed, for example, by R. Resnick and D. Halliday, Physics (Wiley, New York 1967), supplementary topic iv, p. 11; R. Becker and G. Leifried, Theory of Heat 2nd. ed. (Springer, New York 1967), p. 94.

[12] Some aspects of the scalar field case in four spacetime dimensions are given by T. H. Boyer, "Derivation of the Planck spectrum for relativistic classical scalar radiation from thermal equilibrium in an accelerating frame," Phys. Rev. D 81, 105024(10) (2010).

[13] H. Goldstein, Classical Mechanics 2nd ed. (Addison-Wesley, Reading, MA 1981), p. 575-580.

[14] Additional comments on scaling symmetry can be found in T. H. Boyer, "Scaling symmetries of scatterers of classical zero-point radiation," J. Phys. A: Math. Theor. 40, 9635-9642 (2007).

[15] Discussion of random radiation in terms of random phases can be found in the article by S. O. Rice, in Selected papers on Noise and Stochastic Processes, edited by N. Wax (Dover, New York 1954), p. 38.

[16] See for example, ref. 1, p. 339.

[17] This divergence is termed the "infrared divergence" in two-dimensional massless scalar fields in Minkowski spacetime; S. R. Coleman, "There are no Goldstone bosons in two dimensions," Commun. Math. Phys. 31, 259-264 (1973).

[18] S. A. Fulling and P. C. W. Davies, "Radiation from a moving mirror in two dimensional space-time: conformal anomaly," Proc. R. Soc. Lond. A. 348, 393-414 (1976), p. 407.

[19] R. C. Tolman, Thermodynamics and Cosmology (Dover, New York 1987), p. 318: R. C. Tolman and P. Ehrenfest, Phys. Rev. 35, 1791-1978 (1930).

[20] See for example, J. Mathews and R. L. Walker, Mathematical Methods of Physics 2nd ed. (Benjamin/Cummins, Reading, MA 1970), p. 264, 338.

[21] I. S. Gradshteyn and I. M. Ryzhik, Tables of Integrals, Series, and Products (Academic, New 
York, 1965), p. 494, No.12

$$
\int_{0}^{\infty} d x \frac{x^{2 m} \sin b x}{\exp (x)-1}=(-1)^{m} \frac{\partial^{2 m}}{\partial b^{2 m}}\left[\frac{\pi}{2} \operatorname{coth} b \pi-\frac{1}{2 b}\right], \quad b>0
$$

[22] See for example, ref. 2, p. 12.

[23] T. H. Boyer, "Equilibrium of random classical electromagnetic radiation in the presence of a nonrelativistic nonlinear electric dipole oscillator," Phys. Rev. D 13, 2832-2845 (1976); "Statistical equilibrium of nonrelativistic multiply periodic classical systems and random classical electromagnetic radiation," Phys. Rev. A 18, 1228-1237 (1978).

[24] T. H. Boyer, "Blackbody radiation and the scaling symmetry of relativistic classical electron theory with classical electromagnetic zero-point radiation," Found. Phys. 40, 1102-1116 (2010).

[25] P. C. W. Davies, "Scalar particle production in Schwarzschild and Rindler metrics," J. Phys. A: Math. Gen. 18, 609-616 (1975).

[26] See the recent review by L. C. B. Crispino, A. Higuchi, G. E. A. Matsas, "The Unruh effect and its applications," Rev. Mod. Phys. 80, 787-838 (2008).

[27] Connections between the classical and quantum treatments are described by T. H. Boyer, "Classical and quantum interpretations regarding thermal behavior in a coordinate frame accelerating through zero-point radiation," submitted for publication. 